\title{
Einstein A-coefficients for vib-rotational transitions in $\mathrm{CO}^{\star}$
}

\author{
S. Chandra ${ }^{1,2}$, V.U. Maheshwari ${ }^{2}$ and A.K. Sharma ${ }^{1}$ \\ 1 Department of Physics, University of Gorakhpur, Gorakhpur 273 009, India \\ 2 Inter-University Centre for Astronomy and Astrophysics, Post Bag 4, Ganeshkhind, Pune 411007 , India
}

Received May 18; accepted December 1, 1995

\begin{abstract}
Einstein A-coefficients for vib-rotational transitions in CO isotopomers, for vibrational quantum number $v$ up to 20, rotational quantum number $J$ up to 140, and $\Delta v$ up to 4 , are calculated. The change in $J$ is governed by the selection rules $\Delta J= \pm 1$. These coefficients play an important role in astronomy, as CO is the most abundant molecule after $\mathrm{H}_{2}$, and has been observed in almost all the astronomical objects.
\end{abstract}

Key words: ISM: molecules — molecular data

\section{Introduction}

Carbon monoxide has been observed in almost all the astronomical objects and is the most abundant molecule after $\mathrm{H}_{2}$. Hence, it has been a molecule of great interest for astrophysicists. For its theoretical studies, one of the essential parameters is the Einstein A-coefficients for vib-rotational transitions. The Einstein A-coefficients for vib-rotational transitions for $\mathrm{CO}$ have been calculated by a number of authors, e.g., Chackerian \& Tipping (1983), Hure \& Roueff (1993) and Goorvitch \& Chackerian (1994a, b). The reason, for recalculations for these coefficients has been either more accurate Dunham coefficients, derived from the accurate and large number of transitions in the higher vibrational states, were obtained, or more accurate electric dipole moment function (EDMF) from the experimental measurements or ab-initio calculations was obtained. Chackerian \& Tipping (1983) used the Dunham coefficients derived by Dale et al. (1979), and the EDMF, used by them, was obtained by using the data from various accurate observations. Hure \& Roueff (1993) used the energies which were in agreement often better than 0.01 $\mathrm{cm}^{-1}$ with the laboratory data of Farrenq et al. (1991), Le Floch (1991a, b), Guelachvili et al. (1983), and the EDMF was that calculated by Cooper \& Kirby (1987). Goorvich \& Chackerian (1994a, b) used the Dunham coefficients of Farrenq et al. (1991) and the EDMF experimentally derived by Chackerian et al. (1984).

Besides the accuracy of the Dunham coefficients and the EDMF, one more important aspect in the calcula-

Send offprint requests to: S. Chandra, School of Sciences, Indira Gandhi National Open University, New Delhi 110 068, India

${ }^{\star}$ Tables 2A, 2B, 2C and 2D are available only in electronic form via anonymous ftp 130.79.128.5 at the CDS tions is the accurate determination of the potential energy curve by using the values of the Dunham coefficients. Our (Chandra et al. 1995) recent calculations for the Einstein A-coefficients for the CS molecule showed that the Einstein A-coefficients, particularly for large $J$ and/or large $v$ are very sensitive to the potential energy curve. Various methods for determination of the potential energy curve have been suggested time to time (see, e.g., Chandra \& Sharma 1995). Recently, Chandra \& Maheshwari (1995), and Chandra \& Sharma (1995) have proposed new methods for the calculation of the potential energy curve. These methods are very fast and the accuracy of the turning points in the potential energy curve is at least $10^{-7} \AA$, which is better than that of the other methods, used earlier.

In the present investigation, we have calculated Einstein A-coefficients for the vib-rotational transitions in the more abundant CO isotopomers. We have used the Dunham coefficient of Farrenq et al. (1991) and the EDMF of Chackerian et al. (1984). However, the potential energy curve, to which the calculations are more sensitive than other parameters, is calculated by using the methods of Chandra \& Maheshwari (1995), and Chandra \& Sharma (1995). Both of these methods produce the turning points of the same accuracy of at least $10^{-7} \AA$. The Dunham coefficients of Farrenq et al. (1991) are very accurate as these have been derived from the laboratory data available up to very high vibrational states $(v \leq 41)$.

\section{Method of calculation and results}

The RKR potential for the molecule $\mathrm{CO}$ has been calculated with the help of the methods of Chandra \& Maheshwari (1995), and Chandra \& Sharma (1995) where the Dunham coefficients of Farrenq et al. (1991) are used. 
Table 1. Turning points (in $\AA$ ) and potential energy $\left(\right.$ in $\mathrm{cm}^{-1}$ ) for ${ }^{12} \mathrm{C}^{16} \mathrm{O}$

\begin{tabular}{|r|ccr||r|ccc|}
\hline$v$ & $r_{\min }$ & $r_{\max }$ & pot. energy & $v$ & $r_{\min }$ & $r_{\max }$ & pot. energy \\
\hline- & $R_{e}$ & $R_{s}$ & 0.0000 & 20 & 0.9115891 & 1.5960224 & 38998.6766 \\
0 & 1.0833117 & 1.1787672 & 1081.5857 & 21 & 0.9081211 & 1.6134756 & 40626.6001 \\
1 & 1.0534226 & 1.2196100 & 3224.8568 & 22 & 0.9047982 & 1.6309782 & 42229.6144 \\
2 & 1.0342213 & 1.2498865 & 5341.6478 & 23 & 0.9016101 & 1.6485461 & 43807.8009 \\
3 & 1.0193930 & 1.2759139 & 7432.0247 & 24 & 0.8985475 & 1.6661943 & 45361.2396 \\
4 & 1.0071103 & 1.2995223 & 9496.0550 & 25 & 0.8956024 & 1.6839371 & 46890.0081 \\
5 & 0.9965406 & 1.3215471 & 11533.8078 & 26 & 0.8927673 & 1.7017887 & 48394.1814 \\
6 & 0.9872218 & 1.3424533 & 13545.3543 & 27 & 0.8900356 & 1.7197625 & 49873.8318 \\
7 & 0.9788664 & 1.3625314 & 15530.7670 & 28 & 0.8874012 & 1.7378720 & 51329.0276 \\
8 & 0.9712811 & 1.3819774 & 17490.1202 & 29 & 0.8848588 & 1.7561305 & 52759.8334 \\
9 & 0.9643289 & 1.4009315 & 19423.4901 & 30 & 0.8824033 & 1.7745513 & 54166.3090 \\
10 & 0.9579086 & 1.4194984 & 21330.9539 & 31 & 0.8800304 & 1.7931478 & 55548.5091 \\
11 & 0.9519425 & 1.4377586 & 23212.5907 & 32 & 0.8777358 & 1.8119337 & 56906.4825 \\
12 & 0.9463702 & 1.4557764 & 25068.4807 & 33 & 0.8755161 & 1.8309228 & 58240.2716 \\
13 & 0.9411431 & 1.4736037 & 26898.7056 & 34 & 0.8733677 & 1.8501297 & 59549.9117 \\
14 & 0.9362215 & 1.4912839 & 28703.3478 & 35 & 0.8712878 & 1.8695691 & 60835.4299 \\
15 & 0.9315727 & 1.5088537 & 30482.4908 & 36 & 0.8692735 & 1.8892565 & 62096.8449 \\
16 & 0.9271692 & 1.5263443 & 32236.2189 & 37 & 0.8673224 & 1.9092080 & 63334.1657 \\
17 & 0.9229877 & 1.5437834 & 33964.6167 & 38 & 0.8654323 & 1.9294409 & 64547.3909 \\
18 & 0.9190083 & 1.5611953 & 35667.7691 & 39 & 0.8636015 & 1.9499730 & 65736.5076 \\
19 & 0.9152138 & 1.5786016 & 37345.7608 & 40 & 0.8618281 & 1.9708235 & 66901.4905 \\
\hline
\end{tabular}

The turning points $\left(r_{\min }\right.$ and $\left.r_{\max }\right)$ in the potential energy curve and the corresponding potential energy for ${ }^{12} \mathrm{C}^{16} \mathrm{O}$ are given in Table 1. By using this potential energy curve, the Einstein A-coefficients are calculated with the help of the program LEVEL (Le Roy 1993). In the calculations, we used the value of the equilibrium nuclear separation, $R_{\mathrm{e}}=1.1283226$ Åand the dissociation energy $90674 \mathrm{~cm}^{-1}$ (Eidelsberg et al. 1987).

We calculated the A-coefficients for vibrational quantum number $v$ up to 20 , rotational quantum number $J$ up 140 , and $\Delta v$ up to 4 . The numerical results are given in Tables 2A, 2B, 2C and 2D for ${ }^{12} \mathrm{C}^{16} \mathrm{O},{ }^{12} \mathrm{C}^{17} \mathrm{O},{ }^{12} \mathrm{C}^{18} \mathrm{O}$ and ${ }^{13} \mathrm{C}^{16} \mathrm{O}$, respectively, and are available in electronic form via anonymous ftp 130.79.128.5 at the CDS. In the Tables $V L$ and $J L$ are, respectively, the vibrational and rotational quantum numbers for the lower level, and $V U$ and $J U$ are the corresponding numbers for the upper level for a transition. The units of the Einstein A-coefficient, $A$, energy of the lower level, $E$ and the frequency of the transition, freq, are $\mathrm{s}^{-1}, \mathrm{~cm}^{-1}$ and $\mathrm{cm}^{-1}$, respectively. A part of the Table $2 \mathrm{~A}$ for $v$ up to 2 and $J$ up to 10 is given, here, in the printed form.
Acknowledgements. This work was started when one of the authors (SC) visited the group of Prof. Dr. W.H. Kegel under the bilateral scientific exchange programme between the I.N.S.A., New Delhi and the D.F.G., Bonn. He is grateful to Prof. Kegel and the members of his group, especially to Ms. A. Tüpker, for their warm hospitality. We are grateful to Prof. J.V. Narlikar for his encouragements.

\section{References}

Chandra S., Kegel W.H., Le Roy R.J., Hertenstein T., 1995, A\&AS 114, 175

Chandra S., Maheshwari V.U., 1995 (submitted)

Chandra S., Sharma A.K., 1995 (submitted)

Chackerian C., Farrenq R., Guelachvili G., Rossetti C., Urban W., 1984, Can. J. Phys. 62, 1579

Chackerian C., Tipping R.H., 1983, J. Mol. Spectrosc. 99, 431

Cooper D.L., Kirby K., 1987, J. Chem. Phys. 87, 424

Dale R.M., Herman M., Johns J.W.C., et al., 1979, Can. J. Phys. 57, 667

Eidelsberg M., Roncin J.-Y., Le Floch A., et al., 1987, J. Mol. Spectrosc. 121, 309

Farrenq R., Guelachvili G., Sauval A.J., Grevesse N., Farmer C.B., 1991, J. Mol. Spectrosc. 149, 375 
Table 2. A. Einstein A-coefficients, energy of lower level, and frequency of transition for $\mathrm{C}^{12} \mathrm{O}^{16}$

\begin{tabular}{|c|c|c|c|c|c|c|c|c|c|c|c|c|c|}
\hline VI & $\mathrm{JL}$ & VU & $\mathrm{JU}$ & A(Eins) & $\mathrm{E}$ (lower) & freq & VL & JL & VU & JU & $A($ Eins $)$ & E(lower) & freq \\
\hline 0 & 0 & 0 & 1 & $7.67 \bar{D}-08$ & 1081.5857 & 3.8450 & 0 & 1 & 0 & 2 & $7.36 \mathrm{D}-07$ & 1085.4307 & 7.6899 \\
\hline 0 & 2 & 0 & 3 & $2.66 \mathrm{D}-06$ & 1093.1206 & 11.5345 & 0 & 3 & 0 & 4 & $6.53 \mathrm{D}-06$ & 1104.6551 & 15.3787 \\
\hline 0 & 4 & 0 & 5 & $1.30 \mathrm{D}-05$ & 1120.0338 & 19.2222 & 0 & 5 & 0 & 6 & $2.28 \mathrm{D}-05$ & 1139.2560 & 23.0651 \\
\hline 0 & 6 & 0 & 7 & $3.65 \mathrm{D}-05$ & 1162.3211 & 26.9070 & 0 & 7 & 0 & 8 & $5.47 \mathrm{D}-05$ & 1189.2281 & 30.7479 \\
\hline 0 & 8 & 0 & 9 & $7.81 \mathrm{D}-05$ & 1219.9760 & 34.5877 & 0 & 9 & 0 & 10 & $1.07 \mathrm{D}-04$ & 1254.5637 & 38.4261 \\
\hline 0 & 1 & 1 & 0 & $3.42 \mathrm{D}+01$ & 1085.4307 & 2139.4261 & 0 & 0 & 1 & 1 & $1.15 \mathrm{D}+01$ & 1081.5857 & 2147.0811 \\
\hline 0 & 2 & 1 & 1 & $2.26 \mathrm{D}+01$ & 1093.1206 & 2135.5462 & 1 & 0 & 1 & 1 & 4.90D-08 & 3224.8568 & 3.8100 \\
\hline 0 & 1 & 1 & 2 & $1.39 \mathrm{D}+01$ & 1085.4307 & 2150.8560 & 0 & 3 & 1 & 2 & $2.03 \mathrm{D}+01$ & 1104.6551 & 2131.6316 \\
\hline 1 & 1 & 1 & 2 & $4.70 \mathrm{D}-07$ & 3228.6668 & 7.6199 & 0 & 2 & 1 & 3 & $1.50 \mathrm{D}+01$ & 1093.1206 & 2154.5956 \\
\hline 0 & 4 & 1 & 3 & $1.92 \mathrm{D}+01$ & 1120.0338 & 2127.6824 & 1 & 2 & 1 & 3 & $1.70 \mathrm{D}-06$ & 3236.2867 & 11.4295 \\
\hline 0 & 3 & 1 & 4 & $1.56 \mathrm{D}+01$ & 1104.6551 & 2158.2997 & 0 & 5 & 1 & 4 & $1.85 \mathrm{D}+01$ & 1139.2560 & 2123.6988 \\
\hline 1 & 3 & 1 & 4 & $4.16 \mathrm{D}-06$ & 3247.7162 & 15.2386 & 0 & 4 & 1 & 5 & $1.60 \mathrm{D}+01$ & 1120.0338 & 2161.9682 \\
\hline 0 & 6 & 1 & 5 & $1.81 \mathrm{D}+01$ & 1162.3211 & 2119.6810 & 1 & 4 & 1 & 5 & 8.29D-06 & 3262.9548 & 19.0472 \\
\hline 0 & 5 & 1 & 6 & $1.64 \mathrm{D}+01$ & 1139.2560 & 2165.6010 & 0 & 7 & 1 & 6 & $1.78 \mathrm{D}+01$ & 1189.2281 & 2115.6290 \\
\hline 1 & 5 & 1 & 6 & $1.45 \mathrm{D}-05$ & 3282.0020 & 22.8550 & 0 & 6 & 1 & 7 & $1.66 \mathrm{D}+01$ & 1162.3211 & 2169.1979 \\
\hline 0 & 8 & 1 & 7 & $1.75 \mathrm{D}+01$ & 1219.9760 & 2111.5430 & 1 & 6 & 1 & 7 & 2.32D-05 & 3304.8571 & 26.6620 \\
\hline 0 & 7 & 1 & 8 & $1.69 \mathrm{D}+01$ & 1189.2281 & 2172.7588 & 0 & 9 & 1 & 8 & $1.73 \mathrm{D}+01$ & 1254.5637 & 2107.4232 \\
\hline 1 & 7 & 1 & 8 & $3.48 \mathrm{D}-05$ & 3331.5190 & 30.4679 & 0 & 8 & 1 & 9 & $1.71 D+01$ & 1219.9760 & 2176.2835 \\
\hline 0 & 10 & 1 & 9 & $1.71 \mathrm{D}+01$ & 1292.9898 & 2103.2697 & 1 & 8 & 1 & 9 & 4.96D-0.5 & 3361.9869 & 34.2726 \\
\hline 0 & 9 & 1 & 10 & $1.72 \mathrm{D}+01$ & 1254.5637 & 2179.7719 & 1 & 9 & 1 & 10 & $6.80 \mathrm{D}-05$ & 3396.2595 & 38.0760 \\
\hline 0 & 1 & 2 & 0 & 8.34D-01 & 1085.4307 & 4256.2171 & 1 & 1 & 2 & 0 & $6.66 \mathrm{D}+01$ & 3228.6668 & 2112.9810 \\
\hline 0 & 0 & 2 & 1 & $2.83 \mathrm{D}-01$ & 1081.5857 & 4263.8372 & 0 & 2 & 2 & 1 & $5.52 \mathrm{D}-01$ & 1093.1206 & 4252.3022 \\
\hline 1 & 0 & 2 & 1 & $2.24 \mathrm{D}+01$ & 3224.8568 & 2120.5661 & 1 & 2 & 2 & 1 & $4.41 \mathrm{D}+01$ & 3236.2867 & 2109.1361 \\
\hline 2 & 0 & 2 & 1 & $2.18 \mathrm{D}-08$ & 5341.6478 & 3.7750 & 0 & 1 & 2 & 2 & 3.42D-01 & 1085.4307 & 4267.5421 \\
\hline 0 & 3 & 2 & 2 & 4.93D-01 & 1104.6551 & 4248.3176 & 1 & 1 & 2 & 2 & $2.71 \mathrm{D}+01$ & 3228.6668 & 2124.3060 \\
\hline 1 & 3 & 2 & 2 & $3.95 \mathrm{D}+01$ & 3247.7162 & 2105.2566 & 2 & 1 & 2 & 2 & 2.09D-07 & 5345.4229 & 7.5499 \\
\hline 0 & 2 & 2 & 3 & 3.70D-01 & 1093.1206 & 4271.1766 & 0 & 4 & 2 & 3 & $4.66 \mathrm{D}-01$ & 1120.0338 & 4244.2634 \\
\hline 1 & 2 & 2 & 3 & $2.92 \mathrm{D}+01$ & 3236.2867 & 2128.0105 & 1 & 4 & 2 & 3 & $3.74 \mathrm{D}+01$ & 3262.9548 & 2101.3424 \\
\hline 2 & 2 & 2 & 3 & $7.53 \mathrm{D}-07$ & 5352.9728 & 11,3245 & 0 & 3 & 2 & 4 & 3.87D-01 & 1104.6551 & 4274.7407 \\
\hline 0 & 5 & 2 & 4 & 4.49D-01 & 1139.2560 & 4240.1398 & 1 & 3 & 2 & 4 & $3.04 \mathrm{D}+01$ & 3247.7162 & 2131.6797 \\
\hline 1 & 5 & 2 & 4 & $3.62 \mathrm{D}+01$ & 3282.0020 & 2097.3938 & 2 & 3 & 2 & 4 & $1.85 \mathrm{D}-06$ & 5364.2972 & 15.0986 \\
\hline 0 & 4 & 2 & 5 & $3.99 \mathrm{D}-01$ & 1120.0338 & 4278.2342 & 0 & 6 & 2 & 5 & $4.38 \mathrm{D}-01$ & 1162.3211 & 4235.9470 \\
\hline 1 & 4 & 2 & 5 & $3.13 \mathrm{D}+01$ & 3262.9548 & 2135.3132 & 1 & 6 & 2 & 5 & $3.53 \mathrm{D}+01$ & 3304.8571 & 2093.4110 \\
\hline 2 & 4 & 2 & 5 & $3.68 D-06$ & 5379.3959 & 18.8722 & 0 & 5 & 2 & 6 & 4.09D-81 & 1139.2560 & 4281.6570 \\
\hline 0 & 7 & 2 & 6 & 4.29D-01 & 1189.2281 & 4231.6850 & 1 & 5 & 2 & 6 & $3.19 \mathrm{D}+01$ & 3282.0020 & 2138.9110 \\
\hline 1 & 7 & 2 & 6 & $3.46 \mathrm{D}+01$ & 3331.5190 & 2089.3940 & 2 & 5 & 2 & 6 & 6.42D-06 & 5398.2681 & 22.6450 \\
\hline 0 & 6 & 2 & 7 & $4.17 \mathrm{D}-01$ & 1162.3211 & 4285.0089 & 0 & 8 & 2 & 7 & $4.22 \mathrm{D}-01$ & 1219.9760 & 4227.3540 \\
\hline 1 & 6 & 2 & 7 & $3.24 D+01$ & 3304.8571 & 2142.4729 & 1 & 8 & 2 & 7 & $3.41 \mathrm{D}+01$ & 3361.9869 & 2085.3431 \\
\hline 2 & 6 & 2 & 7 & 1.03D-05 & 5420.9131 & 26.4169 & 0 & 7 & 2 & 8 & 4.24D-01 & 1189.2281 & 4288.2898 \\
\hline 0 & 9 & 2 & 8 & 4.16D-01 & 1254.5637 & 4222.9542 & 1 & 7 & 2 & 8 & $3.29 \mathrm{D}+01$ & 3331.5190 & 2145.9988 \\
\hline 1 & 9 & 2 & 8 & $3.37 \mathrm{D}+01$ & 3396.2595 & 2081.2583 & 2 & 7 & 2 & 8 & $1.53 \mathrm{D}-05$ & 5447,3300 & 30.1879 \\
\hline 0 & 8 & 2 & 9 & 4.31D-01 & 1219.9760 & 4291.4994 & 0 & 10 & 2 & 9 & 4.10D-01 & 1292.9898 & $4218.48 \check{7} 7$ \\
\hline 1 & 8 & 2 & 9 & $3.33 \mathrm{D}+01$ & 3361.9869 & 2149.4885 & 1 & 10 & 2 & 9 & $3.33 D+01$ & 3434.3356 & 2077.1399 \\
\hline 2 & 8 & 2 & 9 & $2.18 \mathrm{D}-05$ & 5477.5179 & 33.9576 & 0 & 9 & 2 & 10 & $4.38 \mathrm{D}-01$ & 1254.5637 & 4294.6378 \\
\hline 1 & 9 & 2 & 10 & $3.36 \mathrm{D}+01$ & 3396.2595 & 2152.9419 & 2 & 9 & 2 & 10 & $2.99 \mathrm{D}-05$ & 5511.4755 & 37.7260 \\
\hline
\end{tabular}

Goorvitch D., Chackerian C., 1994a, ApJS 91, 483

Goorvitch D., Chackerian C., 1994b, ApJS 92, 311

Guelachvili G., De Villeneuve D., Farrenq R., Urban W.,

Verges J., 1983, J. Mol. Spectrosc. 98, 64

Hure J.M., Roueff E., 1993, J. Mol. Spectrosc. 160, 335
Le Floch A., 1991a, Mol. Phys. 72, 133

Le Floch A., 1991b, A\&AS 90, 513

Le Roy R.J., 1993, Univ. of Waterloo, Chemical Physics Research Report CP-330R ${ }^{2}$ 